Pacific Journal of Mathematics

ON THE INTEGRAL MEANS OF UNIVALENT, MEROMORPHIC 


\title{
ON THE INTEGRAL MEANS OF UNIVALENT, MEROMORPHIC FUNCTIONS
}

\author{
Albert E. Livingston
}

\begin{abstract}
We consider two classes of functions, univalent and meromorphic in the unit disk $\Delta$. The first class is normalized by requiring that the functions be nonzero in $\Delta$ with $f(0)=1$ and a pole at a fixed point, $p, 0<p<1$. In the second class the functions are allowed to have a zero with fixed magnitude. Theorems concerning the integral means of functions in both classes are proven and consequences of these theorems are considered.
\end{abstract}

1. Introduction. Let $\Sigma(p), 0<p<1$, be the class of functions $f(z)$, univalent and meromorphic in $\Delta=\{z:|z|<1\}$, with a simple pole at $z=p$ and such that $f(z) \neq 0$ for $z$ in $\Delta$ and $f(0)=1$. Also, if $0<p<1$ and $0<q<1$, we let $\Sigma(p, q)$ be the class of functions $f(z)$, univalent and meromorphic in $\Delta$, with a simple pole at $z=p$ such that $f\left(z_{0}\right)=0$ for some $z_{0}$ with $\left|z_{0}\right|=q$ and $f(0)=1$. Recently Libera and the author [4] and the author [5] have studied a subclass of $\Sigma(p)$, namely the class of weakly starlike meromorphic functions $\Lambda^{*}(p)$ which have the representation

$$
f(z)=\frac{z}{\left(1-\frac{z}{p}\right)(1-p z)} g(z)
$$

where $g(z)$ is in $\Sigma^{*}$, the class of normalized meromorphic starlike functions. In this paper we will extend many of the results obtained for $\Lambda^{*}(p)$ to the class $\Sigma(p)$. In particular it was proven in [5] that if $f$ is in $A^{*}(p)$ and $F(z)=(1+z)^{2} /(1-z / p)(1-p z)$, then

$$
\int_{-\pi}^{\pi}\left|f\left(r e^{i \theta}\right)\right|^{\lambda} d \theta \leqq \int_{-\pi}^{\pi}\left|F\left(r e^{i \theta}\right)\right|^{2} d \theta
$$

for $0<r<1$ and $\lambda>0$. Using a powerful method of Baernstein [1], we will extend and generalize this result to the class $\Sigma(p)$. Similar results are also obtained for the class $\Sigma(p, q)$.

2. The class $\Sigma(p)$. The proof of the theorem concerning the integral means of a function in $\Sigma(p)$ follows the proof given by Kirwan and Schober [3] who consider the class $S(p)$ of functions $f(z)$, univalent and meromorphic in $\Delta$, with a simple pole at $z=p$ and such that $f(0)=0$ and $f^{\prime}(0)=1$. The proof relies on results of Baernstein [1] which we now state. 
For this purpose we need to introduce some notation. If $g$ is a measurable, extended real valued function on $[-\pi, \pi]$, then we define

$$
g^{*}(\theta)=\sup _{E} \int_{E} g(\theta) d \theta
$$

where the supremum is taken over all Lebesque measurable sets $E \subset[-\pi, \pi]$ with measure $m(E)=2 \theta$. In particular, if $u\left(r e^{i \theta}\right)$ is defined in an annulus $r_{1}<|z|<r_{2}$ and the $*$ operation is performed in the $\theta$ variable, then $u^{*}\left(r e^{i \theta}\right)$ is defined in $\left\{r e^{i \theta}: r_{1}<r<r_{2}, 0 \leqq \theta \leqq \pi\right\}$. Baernstein [1] has proven the following.

Proposition 1 ([1, Theorems $\mathrm{A}$ and $\mathrm{A}^{\prime}$ and Proposition 5]).

(i) Let $D$ be a domain containing $r_{0}>0$ and having a classical Green's function. Let $u$ be the Green's function of $D$ with pole at $r_{0}$. (It is assumed here that $u$ is defined on the extended plane by defining it to be zero on the complement of $D$.) Then

$$
u^{\sharp}\left(r e^{i \theta}\right)=u^{*}\left(r e^{i \theta}\right)+2 \pi \log ^{+} \frac{r}{r_{0}}
$$

is subharmonic in the upper half-plane.

(ii) Let $D$ and $u$ be as in (i) and suppose further that $D$ is circularly symmetric. Let $D^{+}=D \cap\{z: \operatorname{Im} z>0\}$. Then $u^{\sharp}\left(r e^{i \theta}\right)$ is harmonic in $D^{+}$.

Proposition 2 ([1, Proposition 2]). For $g \in L^{1}[-\pi, \pi]$,

$$
g^{*}(\theta)=\int_{-\theta}^{\theta} G(x) d x, \quad 0 \leqq \theta \leqq \pi,
$$

where $G(x)$ is the symmetric nonincreasing rearrangement of $g$. (For the definition of $G(x)$ see [1] and [2].)

Proposition 3 ([1, Proposition 3]). For $g, h \in L^{1}[-\pi, \pi]$ the following are equivalent.

(a) For every convex nondecreasing function $\Phi$ on $(-\infty, \infty)$,

$$
\int_{-\pi}^{\pi} \Phi(g(\theta)) d \theta \leqq \int_{-\pi}^{\pi} \Phi(h(\theta)) d \theta
$$

(b) For every $t \in(-\infty, \infty)$,

$$
\int_{-\pi}^{\pi}[g(\theta)-t]^{+} d \theta \leqq \int_{-\pi}^{\pi}[h(\theta)-t]^{+} d \theta .
$$

( c ) $g^{*}(\theta) \leqq h^{*}(\theta), 0 \leqq \theta \leqq \pi$. 
We can now state and prove the following theorem.

THEOREM 1. Let $\Phi$ be a convex nondecreasing function on $(-\infty, \infty)$. Then for all $f \in \Sigma(p)$ and $0<r<1$,

$$
\int_{-\pi}^{\pi} \Phi\left( \pm \log \left|f\left(r e^{i \theta}\right)\right|\right) d \theta \leqq \int_{-\pi}^{\pi} \Phi\left( \pm \log \left|F_{p}\left(r e^{i \theta}\right)\right|\right) d \theta
$$

where

$$
F_{p}(z)=\frac{(1+z)^{2}}{\left(1-\frac{z}{p}\right)(1-p z)} .
$$

Proof. We first consider the inequality.

$$
\int_{-\pi}^{\pi} \Phi\left(\log \left|f\left(r e^{i \theta}\right)\right|\right) d \theta \leqq \int_{-\pi}^{\pi} \Phi\left(\log \left|F_{p}\left(r e^{i \theta}\right)\right|\right) d \theta .
$$

With $f^{-1}$ denoting the inverse function of $f$ we define

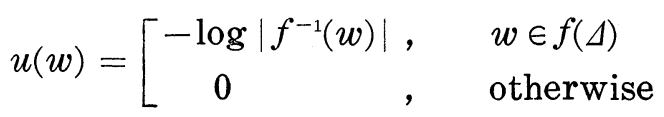

and

$$
v(w)=\left[\begin{array}{cl}
-\log \left|F_{p}^{-1}(w)\right|, & w \in F_{p}(\Delta) \\
0 \quad, & \text { otherwise } .
\end{array}\right.
$$

According to Proposition 1(i) the function $u^{\sharp}\left(r e^{i \theta}\right)=u^{*}\left(r e^{i \theta}\right)+2 \pi \log ^{+} r$ is subharmonic in the upper half-plane and by ([1, Theorem $\left.\left.\mathrm{A}^{\prime}\right]\right)$ is continuous on the real line with 0 deleted. The function $F_{p}$ maps $\Delta$ onto the extended plane slit along the interval $\left[-4 p /(1-p)^{2}, 0\right]$. Thus $F_{p}(\Delta)$ is circularly symmetric and according to Proposition 1(ii) the function $v^{\sharp}\left(r e^{i \theta}\right)=v^{*}\left(r e^{i \theta}\right)+2 \pi \log ^{+} r$ is harmonic in the upper half-plane and by [1, Theorem $\left.\mathrm{A}^{\prime}\right]$ is continuous on the real line with 0 deleted. It follows then that $u^{*}-v^{*}=u^{\sharp}-v^{\sharp}$ is subharmonic in the upper half-plane and continuous on the real line with 0 deleted.

The inequality (2.1) will follow from Proposition $3(b \Rightarrow a)$ if it can be proven that for $f \in \Sigma(p), 0<r<1$ and $0<\rho<\infty$,

$$
\int_{-\pi}^{\pi} \log ^{+} \frac{\left|f\left(r e^{2 \theta}\right)\right|}{\rho} d \theta \leqq \int_{-\pi}^{\pi} \log ^{+} \frac{\left|F_{p}\left(r e^{i \theta}\right)\right|}{\rho} d \theta .
$$

At this point we have need of a lemma analogous to Proposition 4 in [1] and one which appears in [3].

Lemma. Let $f \in \Sigma(p), 0<r<1$ and $0<\rho<\infty$. Then, 
$\int_{-\pi}^{\pi} \log ^{+} \frac{\left|f\left(r e^{i \phi}\right)\right|}{\rho} d \phi+2 \pi \log ^{+} \frac{r}{p}=\int_{-\pi}^{\pi}\left[u\left(\rho e^{i \phi}\right)+\log r\right]^{+} d \phi+2 \pi \log ^{+} \frac{1}{\rho}$.

Because of this Lemma, we see that (2.4) is equivalent to the inequality

$$
\int_{-\pi}^{\pi}\left[u\left(\rho e^{i \phi}\right)+\log r\right]^{+} d \phi \leqq \int_{-\pi}^{\pi}\left[v\left(\rho e^{i \phi}\right)+\log r\right]^{+} d \phi .
$$

However, applying Proposition $3(\mathrm{c} \Rightarrow \mathrm{b})$ we see that (2.5) will hold provided

$$
\left(u^{*}-v^{*}\right)\left(\rho e^{i \phi}\right) \leqq 0, \quad 0<\rho<\infty, \quad 0 \leqq \theta \leqq \pi .
$$

As we have already noted $u^{*}-v^{*}$ is subharmonic in the upper half-plane and continuous on the real line with 0 deleted. In a neighborhood of $w=0$ both $u(w)$ and $v(w)$ are continuous with $u(0)=v(0)=0$. Thus given $\varepsilon>0$ there exists $\delta>0$ such that $|u(w)|<\varepsilon / 2 \pi$ if $|w|<\delta$. Thus if $|w|<\delta, w=\rho e^{i \phi}(0 \leqq \phi \leqq \pi)$ and $m(E)=2 \phi$ we have

$$
\int_{E} u\left(\rho e^{i \theta}\right) d \theta<\frac{\varepsilon}{2 \pi} m(E) \leqq \varepsilon
$$

Therefore

$$
u^{*}\left(\rho e^{i \phi}\right)=\sup _{E} \int_{E} u\left(\rho e^{i \theta}\right) d \theta \leqq \varepsilon .
$$

It follows then that $u^{*}(w)$ approaches 0 as $w$ approaches 0 . A similar statement holds for $v^{*}(w)$. Thus

$$
\lim _{w \rightarrow 0}\left(u^{*}-v^{*}\right)(w)=0 \text {. }
$$

We also have

$$
\lim _{w \rightarrow \infty} u(w)=\lim _{w \rightarrow \infty} v(w)=-\log p .
$$

Thus given $\varepsilon>0$ there exists $\delta>0$ such that $|u(w)+\log p|<\varepsilon / 2 \pi$ and $|v(w)+\log p|<\varepsilon / 2 \pi$ if $|w|>\delta$. Thus if $|w|>\delta, w=\rho e^{i \phi}(0 \leqq$ $\phi \leqq \pi)$ and $m(E)=2 \phi$,

$$
\left|\int_{E}\left(u\left(\rho e^{i \theta}\right)+\log p\right) d \theta\right|<\frac{\varepsilon}{2 \pi} m(E) \leqq \varepsilon .
$$

It follows that

$$
-\varepsilon \leqq u^{*}\left(\rho e^{i \phi}\right)+2 \phi \log p \leqq \varepsilon .
$$

Similarly, we have 


$$
-\varepsilon \leqq v^{*}\left(\rho e^{i \phi}\right)+2 \phi \log p \leqq \varepsilon
$$

Thus

$$
-2 \varepsilon \leqq\left(u^{*}-v^{*}\right)\left(\rho e^{i \phi}\right) \leqq 2 \varepsilon
$$

It follows then that

$$
\lim _{w \rightarrow \infty}\left(u^{*}-v^{*}\right)(w)=0
$$

From (2.8) and previous remarks it follows that the subharmonic function $u^{*}-v^{*}$ is bounded in the upper half-plane. Thus, by the maximum principle, it is enough to prove that $\left(u^{*}-v^{*}\right)(s) \leqq 0$ for $s$ on the real axis $R$.

For this purpose we let

$$
D_{f}=\sup _{w \notin f(\lrcorner)}|w|
$$

and divide the real line into 3 intervals,

$$
R=\left(-\infty,-D_{f}\right) \cup\left[-D_{f}, 0\right) \cup[0,+\infty)
$$

Case (i ). $s \in[0,+\infty)$. Because of (2.7) we need only consider $s \in(0+\infty)$. But then $u^{*}(s)=v^{*}(s)=0$ by definition, if $s>0$.

Case (ii). $s \in\left(-\infty,-D_{f}\right)$. We first note that $u(w)$ is harmonic for $\max \left\{1, D_{f}\right\}<|w| \leqq \infty$ and $v(w)$ is subharmonic in the same region. Thus $(u-v)(w)$ is superharmonic in $\max \left\{1, D_{f}\right\}<|w| \leqq \infty$. In general, $u(w)+\log |w-1|$ is harmonic in $|w|>D_{f}$ and $v(w)+$ $\log |w-1|$ is subharmonic in $|w|>D_{f}$. It follows that $(u-v)(w)$ is superharmonic for $D_{f}<|w| \leqq \infty$. Thus we have

$$
\left(u^{*}-v^{*}\right)(s)=\int_{-\pi}^{\pi}(u-v)\left(|s| e^{i \theta}\right) d \theta \leqq 2 \pi(u-v)(\infty)=0 .
$$

Case (iii). $s \in\left[-D_{f}, 0\right)$. Following Kirwan and Schober [3], for a given $\varepsilon>0$ we introduce the subharmonic function

$$
Q\left(\rho e^{i \phi}\right)=\left(u^{*}-v^{*}\right)\left(\rho e^{i \phi}\right)-\varepsilon \phi \quad(0 \leqq \rho<\infty, 0 \leqq \phi \leqq \pi) .
$$

From previous cases we have,

$$
\lim _{w \rightarrow 8} \sup Q(w) \leqq 0
$$

for all $s \in\left\{R-\left[-D_{f}, 0\right)\right\} \cup\{\infty\}$. Suppose $\sup _{\operatorname{Im} w>0} Q(w)=M>0$. Then as in [3] we have by the maximum principle and (2.9) the existence of some $\hat{s} \in\left[-D_{f}, 0\right)$ such that

$$
Q(\hat{s}) \geqq Q\left(|\hat{s}| e^{i \phi}\right), \quad 0 \leqq \phi \leqq \pi
$$


Thus,

$$
\begin{aligned}
0 & \leqq \lim _{\phi \rightarrow \pi} \frac{Q\left(|\hat{s}| e^{i \phi}\right)-Q(\hat{s})}{\phi-\pi} \\
& =\lim _{\phi \rightarrow \pi} \frac{u^{*}\left(|\hat{s}| e^{i \phi}\right)-u^{*}(\hat{s})}{\phi-\pi}-\lim _{\phi \rightarrow \pi} \frac{v^{*}\left(|\hat{s}| e^{i \phi}\right)-v^{*}(\hat{s})}{\phi-\pi}-\varepsilon .
\end{aligned}
$$

From Proposition 2 and the definition of $G(x)$ [1] it follows that

$$
\lim _{\phi \rightarrow \pi} \frac{u^{*}\left(|\hat{s}| e^{i \phi}\right)-u^{*}(\hat{s})}{\phi-\pi}=2 \min _{0 \leqq \varphi \leqq \pi} u\left(|\hat{s}| e^{i \phi}\right) .
$$

A similar equality holds for $v^{*}$. Combining (2.11) and (2.12) we obtain

$$
0 \leqq 2 \min _{0 \leqq \varphi \leqq \pi} u\left(|\hat{s}| e^{i \phi}\right)-2 \min _{0 \leqq \phi \leqq \pi} v\left(|\hat{s}| e^{i \phi}\right)-\varepsilon \leqq-\varepsilon .
$$

Inequality (2.13) follows since the circle $|w|=|\hat{s}|$ intersects the complement of $f(\Delta)$ and thus $u\left(|\hat{s}| e^{i \phi}\right)=0$ for some $\phi$ and since $v\left(|\hat{s}| e^{i \phi}\right) \geqq 0$ for all $\phi$.

However (2.13) is obviously contradictory and thus we must have $\sup _{\operatorname{Im} w>0} Q(w) \leqq 0$. Letting $\varepsilon \rightarrow 0$ we obtain $\left(u^{*}-v^{*}\right)(s) \leqq 0$ for all $s \in\left[-D_{f}, 0\right)$. This then completes the proof of (2.6) and hence (2.1).

The proof that

$$
\int_{-\pi}^{\pi} \Phi\left(-\log \left|f\left(r e^{i \theta}\right)\right|\right) d \theta \leqq \int_{-\pi}^{\pi} \Phi\left(-\log \left|F_{p}\left(r e^{i \theta}\right)\right|\right) d \theta
$$

follows the proofs given in [1] and [3]. The only difference is that (52) of [1] is replaced by

$$
\int_{-\pi}^{\pi} \log ^{+}\left(\rho\left|f\left(r e^{i \theta}\right)\right|\right) d \theta=2 \pi\left(\log \rho-\log ^{+} \frac{r}{p}\right)+\int_{-\pi}^{\pi} \log ^{+} \frac{1}{\rho\left|f\left(r e^{i \theta}\right)\right|} d \theta .
$$

This then completes the proof of Theorem 1.

We have the following theorem as an immediate consequence of Theorem 1.

THEOREM 2. Let $f \in \Sigma(p)$, then for all $\lambda$, $-\infty<\lambda<\infty$, and $0<r<1$,

$$
\int_{-\pi}^{\pi}\left|f\left(r e^{i \theta}\right)\right|^{2} d \theta \leqq \int_{-\pi}^{\pi}\left|F_{p}\left(r e^{i \theta}\right)\right|^{\lambda} d \theta .
$$

\section{Applications of Theorem 2 .}

THEOREM 3. Let $f \in \Sigma(p)$ and $0<r<1$, then for $|z|=r$.

$$
F_{p}(-r) \leqq|f(z)| \leqq\left|F_{p}(r)\right| \text {. }
$$


REMARK. Inequality (3.1) was obtained earlier by Libera and the author [4] for the class $\Lambda^{*}(p) \subset \Sigma(p)$.

Proof. The right side of (3.1) follows upon taking the $\lambda$ th root of both sides of (2.14) and letting $\lambda \rightarrow+\infty$. To obtain the left side of (3.1) we note that 2.1 gives for $\lambda>0$

$$
\int_{-\pi}^{\pi}\left|\frac{1}{f\left(r e^{i \theta}\right)}\right|^{\lambda} d \theta \leqq \int_{-\pi}^{\pi}\left|\frac{1}{F_{p}\left(r e^{i \theta}\right)}\right|^{\lambda} d \theta
$$

Taking the $\lambda$ th root in the last inequality and letting $\lambda \rightarrow+\infty$ we obtain

$$
\frac{1}{|f(z)|} \leqq \max _{|z|=r} \frac{1}{|f(z)|} \leqq \max _{|z|=r} \frac{1}{\left|F_{p}(z)\right|}=\frac{\left(1+\frac{r}{p}\right)(1+p r)}{(1-r)^{2}}=\frac{1}{F_{p}(-r)} .
$$

The last inequality is equivalent to the left side of (3.1).

Let $f \in \Sigma(p)$ and $f(z)=1+\sum_{n=1}^{\infty} a_{n} z^{n}$ for $|z|<p$. It has been proven [4] that if $f \in \Lambda^{*}(p) \subset \Sigma(p)$, then

$$
\frac{(1-p)^{2}}{p} \leqq\left|\alpha_{1}\right| \leqq \frac{(1+p)^{2}}{p} .
$$

The inequality $\left|a_{1}\right| \leqq(1+p)^{2} / p$ can be obtained for the class $\Sigma(p)$ by considering the case $\lambda=2$ in Theorem 2 and letting $r \rightarrow 0$. However, making use of some results of Kirwan and Schober [3] we can obtain both the upper and lower bounds on $\left|a_{1}\right|$.

TheOREM 4. Let $f \in \Sigma(p)$ and $f(z)=1+\sum_{n=1}^{\infty} a_{n} z^{n}$ for $|z|<p$, then

$$
\frac{(1-p)^{2}}{p} \leqq\left|\alpha_{1}\right| \leqq \frac{(1+p)^{2}}{p}
$$

The inequalities are sharp.

Proof. It is easily seen that if $f \in \Sigma(p)$ with $f^{\prime}(0)=a_{1}$, then we can write $f(z)=a_{1} g(z)+1$ where $g \in S(p)$. According to Kirwan and Schober [3], $g(\Delta)$ contains $\left\{w:|w|<p /(1+p)^{2}\right\}$ and $\left\{w:|w|>p /(1-p)^{2}\right\}$. It follows that $f(\Delta)$ contains

$$
\left\{w:|w-1|<p\left|\alpha_{1}\right| /(1+p)^{2}\right\}
$$

and

$$
\left\{w:|w-1|>p\left|a_{1}\right| /(1-p)^{2}\right\} .
$$


Since $0 \notin f(\Delta)$ we must have $1 \geqq p\left|a_{1}\right| /(1+p)^{2}$ and $1 \leqq p\left|a_{1}\right| /(1-p)^{2}$, which gives (3.2).

The function $F_{p}(z)=(1+z)^{2} /(1-z / p)(1-p z)$ gives equality on the right side of $(3.2)$ and $f(z)=(1-z)^{2} /(1-z / p)(1-p z)$ gives equality on the left side of (3.2).

REMARK. Using Theorem 4 and the representation $f(z)=$ $a_{1} g(z)+1$ where $g \in S(p)$, estimates $\left|a_{n}\right|$ similar to those given in [3] may be obtained. Estimates may also be obtained by using Theorem 2 directly.

In [4] sharp estimates on the quantity $\left|f^{\prime}(z) / f(z)\right|$ were obtained for $f \in A^{*}(p)$. Making use of Theorem 4, we can now extend the results to the class $\Sigma(p)$.

THEOREM 5. Let $f \in \Sigma(p)$ and $w \in \Delta, w \neq p$, then

$$
\frac{1}{\left(1-|w|^{2}\right)} \frac{(1-|a|)^{2}}{|a|} \leqq\left|\frac{f^{\prime}(w)}{f(w)}\right| \leqq \frac{1}{\left(1-|w|^{2}\right)} \frac{(1+|a|)^{2}}{|a|}
$$

where $a=(p-w) /(1-p \bar{w})$.

Moreover, given $w \in \Delta, w \neq p$, there exists a function $f \in \Sigma(p)$ for which equality is obtained on the right side of (3.3) and similarly for the left side of (3.3).

Proof. Let $f \in \Sigma(p)$ and $w \in \Delta, w \neq p$, and let

$$
g(z)=\frac{1}{f(w)} f\left(\frac{e^{i \theta} z+w}{1+\bar{w} e^{i \theta} z}\right)
$$

where $\theta=\arg (p-w) /(1-p \bar{w})$. Obviously $g$ is univalent in $\Delta$ with $g(0)=1$ and letting $a=(p-w) /(1-p \bar{w})$ we see that $g$ has a simple pole at $z=|a|$. Thus $g \in \Sigma(|a|)$. Therefore by Theorem 4 we have

$$
\frac{(1-|a|)^{2}}{|a|} \leqq\left|g^{\prime}(0)\right| \leqq \frac{(1+|a|)^{2}}{|a|} .
$$

A straightforward computation now gives (3.3).

Suppose we are given $w \in \Delta, w \neq p$. Let $a=(p-w) /(1-p \bar{w})$ and $\theta=\arg a$. For $z \in \Delta$, let

$$
f(z)=\frac{\left(1+\frac{w e^{-i \theta}}{|a|}\right)\left(1+|a| w e^{-i \theta}\right)(1+A(z))^{2}}{\left(1-w e^{-i \theta}\right)^{2}\left(1-\frac{A(z)}{|a|}\right)(1-|a| A(z))}
$$


where

$$
A(z)=\frac{z-w}{e^{i \theta}(1-\bar{w} z)}
$$

The function $f(z)$ is univalent in $\Delta$, different from 0 and $f(0)=1$. Moreover, $f$ has a pole at that value of $z$ for which $A(z)=|a|$. That is, when $z=p$. Thus $f \in \Sigma(p)$ and a straightforward computation gives equality on the right side of (3.3).

To obtain sharpness on the left side of (3.3) for a given $w \neq p$, we set

$$
f(z)=\frac{\left(1+\frac{w e^{-i \theta}}{|a|}\right)\left(1+|a| w e^{-i \theta}\right)(1-A(z))^{2}}{\left(1+w e^{-i \theta}\right)^{2}\left(1-\frac{A(z)}{|a|}\right)(1-|a| A(z))}
$$

where $a, \theta$, and $A(z)$ have the same meaning as before. Again it is easily seen that $f \in \Sigma(p)$ and that equality is obtained on the left side of (3.3).

4. The class $\Sigma(p, q)$. In this section we extend the previous results to the class $\Sigma(p, q)$ where the functions now take on the value 0 . Here the function playing the role of $F_{p}(z)$ is the function

$$
\underset{(p, q)}{G(z)}=\frac{\left(1+\frac{z}{q}\right)(1+q z)}{\left(1-\frac{z}{p}\right)(1-p z)} .
$$

It is easily seen that $G_{(p, q)} \in \Sigma(p, q)$ and maps $\Delta$ onto the extended plane slit along the interval

$$
\left[-p(1+q)^{2} / q(1-p)^{2},-p(1-q)^{2} / q(1+p)^{2}\right] \text {. }
$$

THEOREM 6. Let $\Phi$ be a convex nondecreasing function on $(-\infty, \infty)$. Then for all $f \in \Sigma(p, q)$ and $0<r<1$,

$$
\int_{-\pi}^{\pi} \Phi\left( \pm \log \left|f\left(r e^{i \theta}\right)\right|\right) d \theta \leqq \int_{-\pi}^{\pi} \Phi\left( \pm \log \left|G_{(p, q)}\left(r e^{i \theta}\right)\right|\right) d \theta
$$

Proof. We first consider the inequality

$$
\int_{-\pi}^{\pi} \Phi\left(\log \left|f\left(r e^{i \theta}\right)\right|\right) d \theta \leqq \int_{-\pi}^{\pi} \Phi\left(\log \left|G_{(p, q)}\left(r e^{i \theta}\right)\right|\right) d \theta
$$

Let

$$
u(w)=\left[\begin{array}{cl}
-\log \left|f^{-1}(w)\right|, & w \in f(\Delta) \\
0, & \text { otherwise }
\end{array}\right.
$$


and

$$
v(w)=\left[\begin{array}{cl}
-\log \left|G_{(p, q)}^{-1}(w)\right|, & w \in G_{(p, q)}(\Delta) \\
0, & \text { otherwise }
\end{array}\right.
$$

Arguing as in Theorem 1, inequality (4.1) will be proven if we can prove that

$$
\left(u^{*}-v^{*}\right)(s) \leqq 0, \quad s \in R .
$$

For this purpose we let

$$
d_{f}=\inf _{w \notin f(\Delta)}|w| \quad \text { and } \quad D_{f}=\sup _{w \notin f(\Delta)}|w|
$$

and

$$
R=\left(-\infty,-D_{f}\right) \cup\left[-D_{f},-d_{f}\right] \cup\left(-d_{f}, 0\right) \cup[0,+\infty) .
$$

Case (i ). $s \in[0,+\infty)$. This case is exactly as in Theorem 1.

Case (ii). $s \in\left(-\infty,-D_{f}\right)$. The argument is the same as the corresponding case in Theorem 1 .

Case (iii). $s \in\left(-d_{f}, 0\right)$. Since $\left\{w:|w|<d_{f}\right\} \subset f(\Delta)$, we have that $u(w)+\log |w-1|$ is harmonic in $|w|<d_{f}$ and $v(w)+\log |w-1|$ is subharmonic in $|w|<d_{f}$. (The term $\log |w-1|$ is only necessary when $1<d$.) It follows that $(u-v)$ is superharmonic for $|w|<d_{f}$ and therefore

$$
\left(u^{*}-v^{*}\right)(s)=\int_{-\pi}^{\pi}(u-v)\left(|s| e^{i \theta}\right) d \theta \leqq 2 \pi(u-v)(0)=0 .
$$

Case (iv). $s\left[-D_{f}-d_{f}\right]$. The argument in this case is the same as the argument given in case (iii) of the proof of Theorem 1.

This then proves (4.2) and hence (4.1).

The inequality

$$
\int_{-\pi}^{\pi} \Phi\left(-\log \left|f\left(r e^{i \theta}\right)\right|\right) d \theta \leqq \int_{-\pi}^{\pi} \Phi\left(-\log \left|G_{(p, q)}\left(r e^{i \theta}\right)\right|\right) d \theta
$$

is obtained as in Theorem 1 except that (52) of [1] is now replaced by

$$
\begin{aligned}
\int_{-\pi}^{\pi} \log ^{+}\left(\rho\left|f\left(r e^{i \theta}\right)\right|\right) d \theta= & 2 \pi\left[\log \rho+\log ^{+} \frac{r}{q}-\log ^{+} \frac{r}{p}\right] \\
& +\int_{-\pi}^{\pi} \log ^{+} \frac{1}{\rho\left|f\left(r e^{i \theta}\right)\right|} d \theta .
\end{aligned}
$$


We have the following as an immediate consequence of Theorem 6.

TheOREM 7. Let $f \in \Sigma(p, q), 0<r<1,-\infty<\lambda<\infty$, then

$$
\int_{-\pi}^{\pi}\left|f\left(r e^{i \theta}\right)\right|^{\lambda} d \theta \leqq \int_{-\pi}^{\pi}\left|G_{(p, q)}\left(r e^{i \theta}\right)\right|^{2} d \theta
$$

5. Applications of Theorem 7. Arguing as in Theorem 3 we obtain the following.

THEOREM 8. Let $f \in \Sigma(p, q)$, then for $|z|=r$.

$$
\left|G_{(p, q)}(-r)\right| \leqq|f(z)| \leqq\left|G_{(p, q)}(r)\right|
$$

THEOREM 9. Let $f \in \Sigma(p, q)$ and $f(z)=1+\sum_{n=1}^{\infty} a_{n} z^{n},|z|<p$, then

$$
\frac{|p-q|(1-p q)}{p q} \leqq\left|a_{1}\right| \leqq \frac{(p+q)(1+p q)}{p q} .
$$

Both inequalities are sharp.

Proof. Let $f \in \Sigma(p, q)$ with $f\left(z_{0}\right)=0$ where $\left|z_{0}\right|=q$. Let $g(z)=$ $(f(z)-1) / \alpha_{1}$, then $g \in S(p)$. We therefore have [3]

$$
\frac{\left|z_{0}\right|}{\left(1+\frac{\left|z_{0}\right|}{p}\right)\left(1+p\left|z_{0}\right|\right)} \leqq\left|g\left(z_{0}\right)\right| \leqq \frac{\left|z_{0}\right|}{\left|1-\frac{\left|z_{0}\right|}{p}\right|\left(1-p\left|z_{0}\right|\right)} .
$$

Since $g\left(z_{0}\right)=-1 / \alpha_{1}$ and $\left|z_{0}\right|=q$, we immediately obtain (5.1).

Equality on the right side of (5.1) is attained by the function $G_{(p, q)}(z)$ and on the left side by the function

$$
f(z)=(1-z / q)(1-q z) /(1-z / p)(1-p z) .
$$

REMARK. The right side of (5.1) could also be obtained by considering the case $\lambda=2$ of Theorem 7 and letting $r$ approach 0 .

REMARK. We may obtain estimates on $\left|a_{n}\right|, n \geqq 2$, by either using the case $\lambda=1$ of Theorem 7 or by using Theorem 9 and the fact that $f(z)=a_{1} g(z)+1$ where $g \in S(p)$ and then using the estimate on the coefficients of a function in $S(p)$ [3].

As an application of Theorem 9 we obtain the following analogue of Theorem 5 .

THEOREM 10. Let $f \in \Sigma(p, q)$ with $f\left(z_{0}\right)=0,\left|z_{0}\right|=q$, then for 
$w \in \Delta, w \neq z_{0}, w \neq p$,

$$
\begin{gathered}
\frac{1}{1-|w|^{2}}\left[\frac{\| a|-| b||(1-|a||b|)}{|a||b|}\right] \leqq\left|\frac{f^{\prime}(w)}{f(w)}\right| \\
\leqq \frac{1}{1-|w|^{2}}\left[\frac{(|a|+|b|)(1+|a||b|)}{|a||b|}\right]
\end{gathered}
$$

where

$$
|a|=\left|\frac{p-w}{1-p \bar{w}}\right| \text { and } \quad|b|=\left|\frac{z_{0}-w}{1-\bar{w} z_{0}}\right|
$$

The left hand side of (5.2) is sharp and the right side is sharp at least for $|w|<q$.

Proof. Let $f \in \Sigma(p, q)$ with $f\left(z_{0}\right)=0,\left|z_{0}\right|=q$. For $w \in \Delta, w \neq p$, $w \neq z_{0}$, let $a=(p-w) /(1-p \vec{w})$ and $\theta=\arg \alpha$. Let

$$
h(z)=\frac{1}{f(w)} f\left[\frac{e^{i \theta} z+w}{1+\bar{w} e^{i \theta} z}\right]
$$

The function $h$ is univalent and meromorphic in $\Delta$ with $h(0)=1$. Moreover $h$ has a pole at $z=|\alpha|$ and $h(z)=0$ when

$$
z=\left(z_{0}-w\right) / e^{i \theta}\left(1-\bar{w} z_{0}\right)=b
$$

Thus $h \in \Sigma(|a|,|b|)$. By Theorem 9 we then have

$$
\frac{\| a|-| b||(1-|a||b|)}{|a||b|} \leqq\left|h^{\prime}(0)\right| \leqq \frac{(|a|+|b|)(1+|a||b|)}{|a||b|}
$$

which gives (5.2).

With $p$ and $q$ fixed let $w \neq p$ be such that $|w|<q$. Let $a=$ $(p-w) /(1-p \bar{w})$ and $\theta=\arg \alpha$. Choose $z_{0}$ with $\left|z_{0}\right|=q$ such that $\left(z_{0}-w\right) / e^{i \theta}\left(1-\bar{w} z_{0}\right)<0$. Such a choice is possible since $|w|<q$. With this choice of $z_{0}$ let $b=\left(z_{0}-w\right) / e^{i \theta}\left(1-\bar{w} z_{0}\right)$ and define

$$
f(z)=\frac{\left(1+\frac{w e^{-i \theta}}{|a|}\right)\left(1+|a| w e^{-i \theta}\right)\left(1+\frac{A(z)}{|b|}\right)(1+|b| A(z))}{\left(1-\frac{w e^{-i \theta}}{|b|}\right)\left(1-|b| w e^{-i \theta}\right)\left(1-\frac{A(z)}{|a|}\right)(1-|a| A(z))}
$$

where

$$
A(z)=\frac{z-w}{e^{i \theta}(1-\bar{w} z)}
$$

The function $f$ is univalent and meromorphic in $\Delta$ with $f(0)=1$. 
Moreover, $f$ has a pole when $A(z)=|a|$, that is when $z=p$. $f$ has a zero when $A(z)=-|b|$. By the choice of $z_{0}$,

$$
A\left(z_{0}\right)=\left(z_{0}-w_{0}\right) / e^{i \theta}\left(1-\bar{w} z_{0}\right)=-|b| \text {. }
$$

Thus $f\left(z_{0}\right)=0$ and $f \in \Sigma(p, q)$. A straightforward calculation gives equality on the right side of (5.2).

For equality on the left side of (5.2), let $|w|<q, w \neq p$ and $a$ and $\theta$ be as before. Choose $z_{\text {n }}$ so that $\left(z_{0}-w\right) / e^{i \theta}\left(1-\bar{w} z_{0}\right)>0$ and set $b=\left(z_{0}-w\right) / e^{i \theta}\left(1-\bar{w} z_{0}\right)$. With this choice of $z_{0}$, let

$$
f(z)=\frac{\left(1+\frac{w e^{-i \theta}}{|a|}\right)\left(1+|a| w e^{-i \theta}\right)\left(1-\frac{A(z)}{|b|}\right)(1-|b| A(z))}{\left(1+\frac{w e^{-i \theta}}{|b|}\right)\left(1+|b| w e^{-i \theta}\right)\left(1-\frac{A(z)}{|a|}\right)(1-|a| A(z))} .
$$

It is easily seen that $f \in \Sigma(p, q)$ and that we get equality on the left side of (5.2).

Suppose $q<r<p . \quad$ Let $a=(p-r) /(1-p r)$ and $b=(q+r) /(1+q r)$ and let

$$
f(z)=\frac{\left(1+\frac{r}{a}\right)(1+a r)\left(1+\frac{A(z)}{b}\right)(1+b A(z))}{\left(1-\frac{r}{b}\right)(1-b r)}
$$

where

$$
A(z)=\frac{z-r}{1-r z}
$$

The function $f$ has a pole at $z=p$ and a zero at $z=-q$. Thus $f \in \Sigma(p, q)$ and a straightforward computation gives equality on the right side of (5.2) when $w=r$.

Let $p$ and $q$ be fixed and $r>0$. Let $a=(p+r) /(1+p r)$ and $b=(q+r) /(1+q r)$ and

$$
f(z)=\frac{\left(1-\frac{r}{a}\right)(1-a r)\left(1-\frac{A(z)}{b}\right)(1-b A(z))}{\left(1-\frac{r}{b}\right)(1-b r)\left(1-\frac{A(z)}{a}\right)(1-a A(z))}
$$

where

$$
A(z)=\frac{z+r}{1+r z}
$$

The function $f$ has a pole at $z=p$ and a zero at $z=q$. Thus $f \in \Sigma(p, q)$ and we get equality on the left side of (5.2) when $w=-r$. 


\section{REFERENCES}

1. A. Baernstein II, Integral means, univalent functions and circular symmetrization, Acta Math., 133 (1974), 139-169.

2. G. H. Hardy, J. E. Littlewood, and G. Póyla, Inequalities, Cambridge Univ. Press, Cambridge, 1959.

3. W. E. Kirwan and Glenn Schober, Extremal problems for meromorphic univalent functions, J. Analyse Math., to be published.

4. R. J. Libera and A. E. Livingston, Weakly starlike meromorphic univalent functions, Trans. Amer. Math. Soc., 202 (1975), 181-191.

5. A. E. Livingston, Weakly starlike meromorphic univalent functions II, Proc. Amer. Math. Soc., 62 (1977), 47-53.

Received October 11, 1976.

UNIVERSITY OF DeLaWARE

NEWARK, DE 19711 


\title{
PACIFIC JOURNAL OF MATHEMATICS
}

\author{
EDITORS
}

RICHARD ARENS (Managing Editor)

University of California

Los Angeles, California 90024

C. W. CurTis

University of Oregon

Eugene, OR 97403

C. C. MOORE

University of California

Berkeley, CA 94720
J. DUgundJI

Department of Mathematics

University of Southern Californı

Los Angeles, California 90007

R. Finn and J. Milgram

Stanford University

Stanford, California 94305

\section{ASSOCIATE EDITORS}

E. F. BECKENBACH

\section{SUPPORTING INSTITUTIONS}

\author{
UNIVERSITY OF BRITISH COLUMBIA \\ CALIFORNIA INSTITUTE OF TECHNOLOGY \\ UNIVERSITY OF CALIFORNIA \\ MONTANA STATE UNIVERSITY \\ UNIVERSITY OF NEVADA, RENO \\ NEW MEXICO STATE UNIVERSITY \\ OREGON STATE UNIVERSITY \\ UNIVERSITY OF OREGON \\ OSAKA UNIVERSITY
}

UNIVERSITY OF SOUTHERN CALIFORNIA

STANFORD UNIVERSITY

UNIVERSITY OF TOKYO

UNIVERSITY OF UTAH

WASHINGTON STATE UNIVERSITY

UNIVERSITY OF WASHINGTON
AMERICAN MATHEMATICAL SOCIETY
NAVAL WEAPONS CENTER 


\section{Pacific Journal of Mathematics \\ Vol. 72 , No. 1 \\ January, 1977}

Kazuo Anzai and Shiro Ishikawa, On common fixed points for several

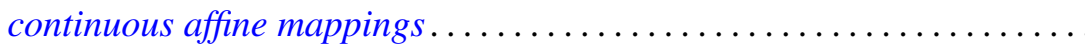

Bruce Alan Barnes, When is a representation of a Banach $*$-algebra

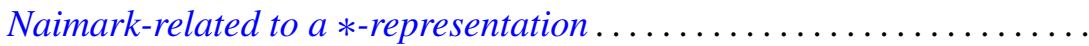

Richard Dowell Byrd, Justin Thomas Lloyd, Franklin D. Pedersen and

James Wilson Stepp, Automorphisms of the semigroup of finite

complexes of a periodic locally cyclic group ...................

Donald S. Coram and Paul Frazier Duvall, Jr., Approximate fibrations and a

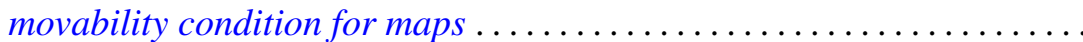

Kenneth R. Davidson and Che-Kao Fong, An operator algebra which is not

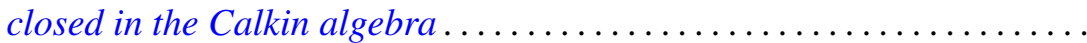

Garret J. Etgen and James Pawlowski, A comparison theorem and oscillation criteria for second order differential systems .............

Philip Palmer Green, $C^{*}$-algebras of transformation groups with smooth

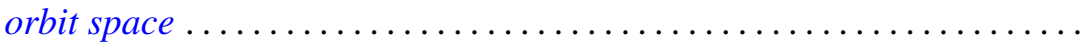

Charles Allen Jones and Charles Dwight Lahr, Weak and norm approximate

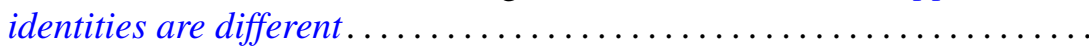

G. K. Kalisch, On integral representations of piecewise holomorphic

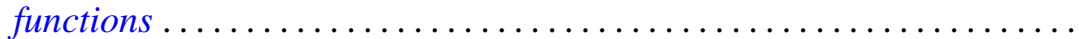

Y. Kodama, On product of shape and a question of Sher

Heinz K. Langer and B. Textorius, On generalized resolvents and

$Q$-functions of symmetric linear relations (subspaces) in Hilbert

space ...................................

Albert Edward Livingston, On the integral means of univalent, meromorphic functions

Wallace Smith Martindale, III and Susan Montgomery, Fixed elements of

Jordan automorphisms of associative rings ..........

R. Kent Nagle, Monotonicity and alternative methods for nonlinear boundary value problems ........................

Richard John O'Malley, Approximately differentiable functions: the $r$ topology.

Mangesh Bhalchandra Rege and Kalathoor Varadarajan, Chain conditions

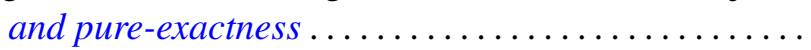

Christine Ann Shannon, The second dual of $C(X)$. .

Sin-ei Takahasi, A characterization for compact central double centralizers of $C^{*}$-algebras .

Theresa Phillips Vaughan, A note on the Jacobi-Perron algorithm. . .

Arthur Anthony Yanushka, A characterization of $\operatorname{PSp}(2 m, q)$ and

$\mathrm{P} \Omega(2 m+1, q)$ as rank 3 permutation groups ......... 